\title{
Mixed-State Ionic Beams: An Effective Tool for Collision Dynamics Investigations
}

\author{
Emmanouil P. Benis ${ }^{1, *(D)}$, Ioannis Madesis ${ }^{2,3}$, Angelos Laoutaris ${ }^{2,3}$, Stefanos Nanos ${ }^{1,3}$ \\ and Theo J. M. Zouros 2,3 (D) \\ 1 Department of Physics, University of Ioannina, GR 45110 Ioannina, Greece; snanos@cc.uoi.gr \\ 2 Department of Physics, University of Crete, Voutes Campus GR 71003 Heraklion, Greece; \\ imadesis@physics.uoc.gr (I.M.); laoutaris@physics.uoc.gr (A.L.); tzouros@physics.uoc.gr (T.J.M.Z.) \\ 3 Tandem Accelerator Laboratory, INPP, NCSR Demokritos, GR 15310 Ag. Paraskevi, Greece \\ * Correspondence: mbenis@uoi.gr; Tel.: +30-26510-08536
}

Received: 29 October 2018; Accepted: 26 November 2018; Published: 29 November 2018

\begin{abstract}
The use of mixed-state ionic beams in collision dynamics investigations is examined. Using high resolution Auger projectile spectroscopy involving He-like $\left(1 s^{2}{ }^{1} S, 1 s 2 s^{3,1} S\right)$ mixed-state beams, the spectrum contributions of the $1 s 2 s^{3} S$ metastable beam component is effectively separated and clearly identified. This is performed with a technique that exploits two independent spectrum measurements under the same collision conditions, but with ions having quite different metastable fractions, judiciously selected by varying the ion beam charge-stripping conditions. Details of the technique are presented together with characteristic examples. In collisions of $4 \mathrm{MeV} \mathrm{B}^{3+}$ with $\mathrm{H}_{2}$ targets, the Auger electron spectrum of the separated $1 s 2 s^{3} S$ boron beam component allows for a detailed analysis of the formation of the $1 s 2 s\left({ }^{3} S\right) n l^{2} L$ states by direct $n l$ transfer. In addition, the production of hollow $2 s 2 p^{1,3} P$ doubly- and $2 s 2 p^{2}{ }^{2} D$ triply-excited states, by direct excitation and transfer-excitation processes, respectively, can also be independently studied. In similar mixed-state beam collisions of $15 \mathrm{MeV} \mathrm{C}^{4+}$ with $\mathrm{H}_{2}, \mathrm{He}, \mathrm{Ne}$ and Ar targets, the contributions of the $1 s^{2}, 1 s 2 s^{3,1} \mathrm{~S}$ beam components to the formation of the $2 s 2 p^{3,1} P$ states by double-excitation, $1 s \rightarrow 2 p$ excitation and transfer-loss processes can be clearly identified, facilitating comparisons with theoretical calculations.
\end{abstract}

Keywords: zero-degree Auger projectile spectroscopy; mixed-state beams; metastable states; He-like states; Li-like states; Be-like states; cascade feeding; electron transfer excitation; electron excitation; electron transfer; hollow states; SIMION

PACS: $34.50 . F a ; 29.27 . F h ; 32.80 . H d ; 32.80 . \mathrm{Dz} ; 34.70 .1 \mathrm{e} ; 34.80 . \mathrm{Kw}$

\section{Introduction}

Over the past four decades considerable attention has been paid to the study of ion-atom collisions using highly charged ion projectiles at various accelerator facilities [1,2]. This interest has been driven primarily by the need for a basic understanding of atomic collisions processes such as electron transfer, excitation and ionization and their combinations which lead to the production of excited states of matter [3-7]. In particular, high resolution studies of projectile ions can provide state-selective information with important practical bearing on controlled thermonuclear fusion, laboratory and astrophysical plasmas, ion beam tumor therapy, as well as the development of new ion sources, the promotion of new and improved accelerator technology and the creation of vuv and x-ray lasers [8,9]. For example, in astrophysical plasma line-intensity ratios are used in modeling plasma dynamics, as well plasma diagnostics $[10,11]$. Existing theories that have successfully predicted total cross-sections for these processes can now be tested to the next order of sophistication 
and accuracy by comparing to state-selective differential cross section measurements. Furthermore, few-electron systems as found on highly charged ions provide some of the simplest testing grounds for studying the many-particle problem at large, and in particular, the role played by electron-electron interactions [12-22]. By limiting the number of electrons on the projectile and by using simple targets such as $\mathrm{He}$ or $\mathrm{H}_{2}$, considerable simplification of the collision system is attained. Thus, the study of these fundamental atomic collision processes, becomes considerably more trackable from both the experimental and the theoretical point of view.

The charge state $q$ of the ionic beam, and therefore the number $N_{p}^{e}$ of electrons $\left(N_{p}^{e}=Z_{p}-q\right)$ and participating shells carried into the collision, can be conveniently selected offering the necessary conditions for initiating and observing specific atomic processes. In general, the lighter the projectile (smaller projectile atomic number $Z_{p}$ ), the fewer the important channels available for ionization, capture or excitation of the projectile. Furthermore, light projectiles also have small fluorescence yields, but much larger Auger electron yields, thus making the use of high resolution Auger projectile spectroscopy, particularly attractive for their investigation [23,24].

In ion accelerators, the extracted ionic beam is usually selected magnetically for a particular charge state $q$ and kinetic energy, as required by the experiment. However, magnetic selection cannot separate the electronic configurations of particular ionic charge states which, due to their particularly long lifetimes, survive to the target resulting in collisions of mixed-state ionic beams consisting of more than just the ground state. A well-known example are the He-like $\left(1 s^{2}{ }^{1} S, 1 s 2 s^{1,3} S\right)$ mixed-state beams. These additional metastable beam components offer the opportunity of studying dynamic collision processes in new ionic environments already having an initial K-shell vacancy. Such pre-excited ionic beams are presently used in collisions with electrons, atoms or photons since they allow for the population of states not readily accessible from the ground state. So far the use of pre-excited long-lived states, has been successfully used in high resolution projectile electron spectroscopy investigations, as for example in single [25-27] and double [28] electron transfer, excitation [29], transfer-excitation [16,30-33], the production of triply-excited states [34] and superelastic scattering $[35,36]$. In addition, long-lived $1 s 2 s^{3} S$ states, have also been used in a variety of other atomic physics investigations including the study of electron impact ionization [37,38], tokamak high energy charge-exchange [39] and edge impurities [40], electron capture and excitation [41], slow collisions of quasi symmetric heavy systems [42], beam-two-foil spectroscopy [43] and even two-electron quantum entanglement [44].

Here, we examine the use of He-like $\left(1 s^{2}{ }^{1} S, 1 s 2 s^{1,3} S\right)$ mixed-state ionic beams and their role in collision dynamics investigations using zero-degree Auger projectile spectroscopy (ZAPS). First, we review the production processes of mixed-state He-like, as well as Be-like ionic beams, the lifetimes of their metastable components and the methods for determining their metastable fractions. Then, we present a method for separating the contributions from the ground and metastable components and report on KLn Auger spectra separated from just the $1 s 2 s^{3} S$ component obtained in collisions $4 \mathrm{MeV}$ $\mathrm{B}^{3+}$ with $\mathrm{H}_{2}$ targets. Finally, we investigate the various processes contributing to the formation of the $2 s 2 p^{1,3} P$ states in collisions of mixed-state $15 \mathrm{MeV} \mathrm{C}^{4+}$ with $\mathrm{H}_{2}, \mathrm{He}, \mathrm{Ne}$ and Ar targets.

\section{Production of Metastable States}

Highly charged ions are readily produced by passing a lower charge state beam through a thin foil or gas, where additional electrons can be stripped from the ion, thus increasing its charge state. In tandem Van de Graaff accelerators, hereafter called TANDEM, these projectile electron strippers are found inside the accelerator terminal, where the initially negatively charged ion beam is converted to a positively charged beam and further energy boosted in the second stage of acceleration. These positively charged ion beam has a Gaussian-like charge state distribution centered around the mean charge state, depending on the energy of the ion beam during the stripping process as well as the stripping medium. The higher the energy of the beam and the density of the medium, the higher the mean charge state attained $[45,46]$. Various computer codes have been developed providing accurate 
results for these charge state distribution such as ETACHA [47], CHARGE [46,48-50] and TARDIS [51] and references therein. The desired charge state and energy are then selected by means of analyzing and switching magnets, as well as the necessary dipole magnetic focusing elements and then delivered to the experimental area. To produce more intense few-electron or even bare ion beams additional stripping points are provided after the beam exits the accelerator known as post-strippers.

A significant operational advantage of the gas strippers is that they do not suffer damage, as do the foils, particularly at the lower stripping energies. Aside from this, the use of gas strippers, as opposed to foils, has certain advantages for high resolution electron spectroscopy in ion-atom collisions. In gas stripping the ions suffer less straggling than in foil stripping [52]. Thus, the gas-stripped ion beams have a narrower energy distribution which is evident in the broadening of the observed projectile Auger lines in high resolution measurements.

Selection of a charge state at a certain energy may result in more than one ionic electron configurations as these cannot be magnetically separated. Indeed, few-electron ionic beams are typically delivered in the ground state and additional long-lived, metastable components. This is a general feature not only encountered in TANDEM accelerators [53,54], but also in storage rings [55,56], as well as other lower energy highly charged ion sources [37], even though the production mechanisms can be quite different depending on the type of ion source. Clearly, a desirable feature would be to have a variable and controllable amount of metastable beam. Such a feature could be used to readily distinguish between ground state and metastable state contributions. This can be readily accomplished in storage rings, where the metastable states can be allowed to die out by storing the ions long enough [56].

As an example, we may refer to the case of He-like ionic beams that are delivered in a mixed $1 s^{2}{ }^{1} \mathrm{~S}$ ground and $1 s 2 s^{3} S$ metastable states (omitting a very small fraction of $1 s 2 s{ }^{1} S$ component surviving at the target area). By performing two different measurements with beams of appreciably different metastable fraction $[57,58]$, the contributions from either the ground state or the metastable state can be extracted [59]. The amount of metastables may vary, and thus be controlled, in the case of the gas stripping depending on the stripping energy [57]. In the case of foil striping, the thickness, as well as the atomic number of the foil affects the metastable fraction [57,60-64]. To our knowledge, there are no available codes that predict the fraction of metastable ions, even though much of the required information is already used to compute the equilibrium charge states [65]. Needless to say, such a code would be extremely useful for researchers.

\section{Lifetimes of Metastable States}

The mixed-state content of the ion beam complicates absolute cross section measurements since the accurate determination of the beam content is also required. However, metastable beams offer access to additional population channels that are not readily available from the ground state beams. For example, the $1 s 2 s 2 p^{4} P_{J}$ state populated in collisions of He-like ionic beams with gas targets is solely populated from the metastable $1 s 2 s^{3} S$ beam component by single electron capture. Population by the $1 s^{2}{ }^{1} S$ component is extremely unlikely as it requires much less effective, higher-order processes.

To fully exploit such metastable components in collision experiments, knowledge of not only the initial metastable fraction content is required, but also the lifetime of the states involved, in order to compute the content at the target. For very long-lived states $(\sim \mathrm{ms})$ this remains unaffected from the production area to the target. However, for metastable states of shorter lifetimes their population at the target may well be affected. Depending on the geometry of the experimental setup the surviving fraction at the target can be estimated, as for example for the He-like beams that are delivered in both $1 s 2 s{ }^{3} S$ and $1 s 2 s{ }^{1} S$ metastable states. The lifetimes of these states have been investigated in the literature and are known to drop rapidly with increasing projectile atomic number $Z_{p}[66,67]$. Similar behavior has been observed for the Be-like $1 s^{2} 2 s 2 p^{3} P$ metastable state also of interest in this work. Indicative theoretical lifetimes for the above states are reproduced in Table 1. 
Table 1. Indicative theoretical lifetimes (in s) of the metastable He-like $1 s 2 s{ }^{1} S, 1 s 2 s^{3} S$ (from Refs. [66,67]) and Be-like $1 s^{2} 2 s 2 p^{3} P_{1}$ states (from Refs. $[68,69]$ ) for $3 \leq Z_{p} \leq 10$.

\begin{tabular}{|c|c|c|c|}
\hline$Z_{p}$ & $1 s 2 s^{1} S$ & $1 s 2 s^{3} S$ & $1 s^{2} 2 s 2 p^{3} P_{1}$ \\
\hline 3 & $5.1 \times 10^{-4}$ & $4.9 \times 10^{1}$ & - \\
\hline 4 & $5.5 \times 10^{-5}$ & $1.8 \times 10^{0}$ & - \\
\hline 5 & $1.1 \times 10^{-5}$ & $1.5 \times 10^{-1}$ & $9.8 \times 10^{-2}$ \\
\hline 6 & $3.0 \times 10^{-6}$ & $2.1 \times 10^{-2}$ & $9.7 \times 10^{-3}$ \\
\hline 7 & $1.1 \times 10^{-6}$ & $3.9 \times 10^{-3}$ & $1.7 \times 10^{-3}$ \\
\hline 8 & $4.3 \times 10^{-7}$ & $9.6 \times 10^{-4}$ & $4.4 \times 10^{-4}$ \\
\hline 9 & $2.0 \times 10^{-7}$ & $2.8 \times 10^{-4}$ & $1.4 \times 10^{-4}$ \\
\hline 10 & $1.0 \times 10^{-7}$ & $9.2 \times 10^{-5}$ & $5.3 \times 10^{-5}$ \\
\hline
\end{tabular}

In Figure 1, we show the surviving fraction of the $1 s 2 s{ }^{1} S$ metastable state as a function of the ion traveling distance $s$ for various low $-Z_{p}$ elements and projectile energies of $0.25-2 \mathrm{MeV} / \mathrm{u}$ typical for a TANDEM accelerator. It should be pointed out that the zero distance $s=0$ may refer to either: (i) the terminal inside the TANDEM tank, where the stripping of the incoming negative ion takes place, or (ii) the post-stripper location when higher charge states are needed. Clearly, the $1 s 2 s{ }^{1} S$ fraction is considerably reduced, even for small distances ( $<<20 \mathrm{~m}$ ), except for beryllium and boron, where larger distances should be considered. Alternatively, the fractions of the $1 s 2 s{ }^{3} S$, as well as the $1 s^{2} 2 s 2 p^{3} P$ metastable states, will remain practically constant over the whole range of $Z_{p}=4-9$ due to their much longer lifetimes.
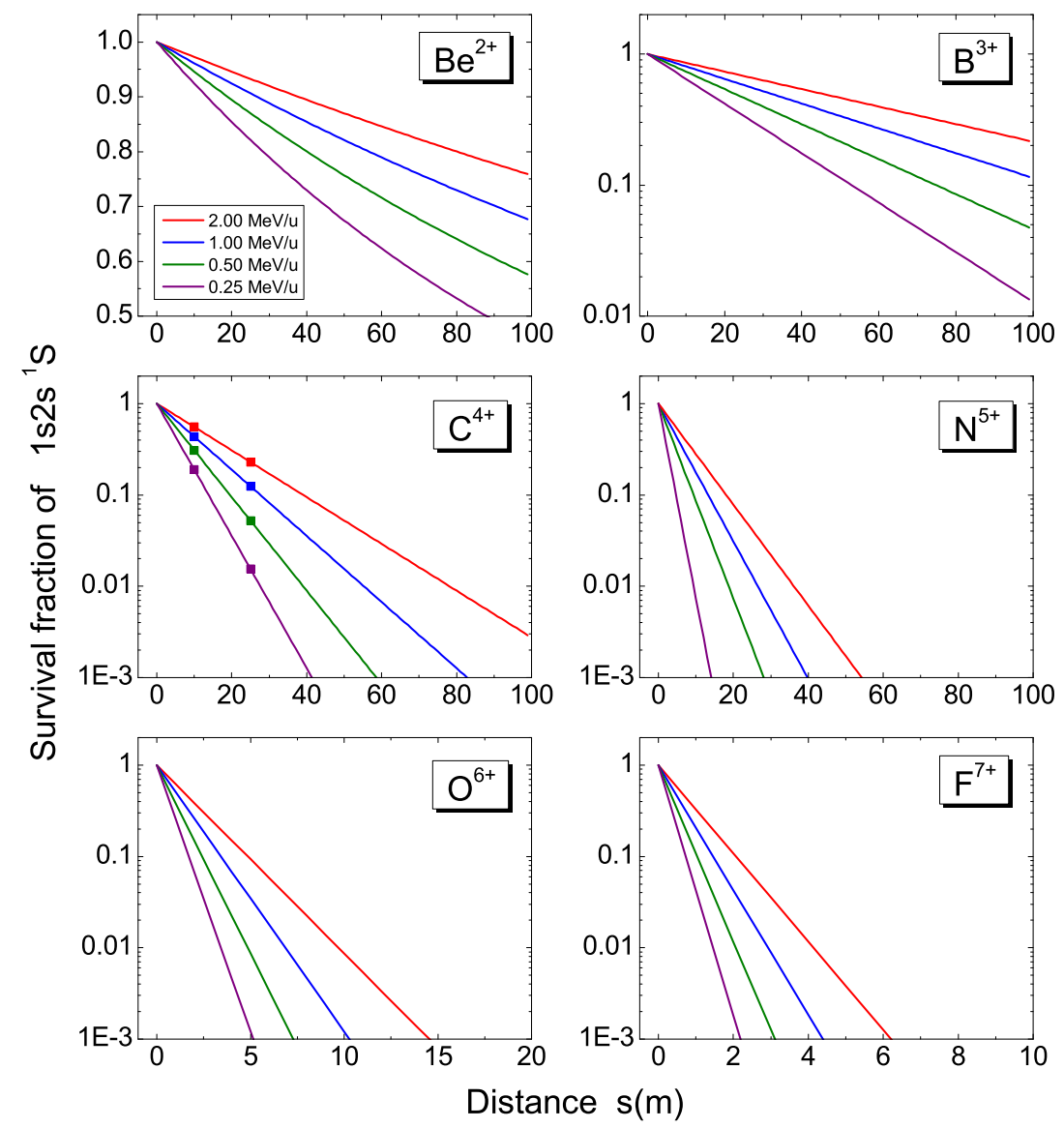

Figure 1. The surviving fraction of the $1 s 2 s{ }^{1} S$ metastable state as a function of the ion beam traveling distance $s$ for various elements with $4 \leq Z_{p} \leq 9$ and typical projectile energies of $0.25-2 \mathrm{MeV} / \mathrm{u}$. Lifetimes are from Ref. [66]. For these He-like ions the survival of the much longer lived $1 s 2 s^{3} S$ metastable state (not shown) is practically $100 \%$ over the same distances. 
For our ZAPS setup, currently operational at the Athens 5.5 MV tandem Van de Graaff accelerator at the National Center for Scientific Research "Demokritos" under the atomic physics with accelerators: projectile electron spectroscopy (APAPES) initiative [70], the interaction region is located at a distance of $s_{1}=25.4 \mathrm{~m}$ from the terminal stripper and of $s_{2}=10 \mathrm{~m}$ from the post-stripper. The surviving fractions of $1 s 2 s^{1} S$ metastable state are indicated in Figure 1 for the case of carbon. In addition, assuming that the $1 s 2 s^{3} S$ and $1 s 2 s^{1} S$ states are statistically produced in a 3:1 ratio, the actual fraction of the $1 s 2 s{ }^{1} S$ beam compared to that of the $1 s 2 s^{3} S$ beam will be further reduced at the production point by a factor of three.

\section{Mixed-State Ionic Beams-Content Determination}

Several techniques can be found in the literature for determining the metastable fraction of multicharged ion beams in various types of facilities. These include: (1) The ion beam attenuation technique [71], where the abundance of excited states in ion beams $\left(25-100 \mathrm{eV} \mathrm{O}^{+}\right.$and $\left.\mathrm{O}^{2+}\right)$ was investigated based on the attenuation of an ion beam during passage from an ion source to a collector through a chamber containing a gas, the pressure of which could be varied, and in which the different states of the ions suffer different attenuations. The metastable fraction is determined by measuring the attenuated ion beam current at distances corresponding to lifetimes of the order of $20 \mu \mathrm{s}$. No information about the configuration of the metastable ionic states was provided. (2) The target K x-ray yield (or beam-foil) technique [60,61], where the metastable fraction of a state is determined based on the ratio of target $\mathrm{K} x$-ray yields from collisions with three different ionic species at various charge states. (3) The photon-particle coincidence technique [72], where the photon from the decay of the $1 s 2 s^{3} S$ metastable state of a neutralized $\mathrm{He}^{+}$ion beam was detected in coincidence with the subsequently singly ionized ion beam. (4) The comparison to similar experimental measurements. For example, normalization to the corresponding ground-state Auger electron spectra [73] or normalization between photoinization and dielectronic recombination cross sections through the detailed balanced principle [74]. (5) The measurement of relative Auger electron yields from doubly excited Li-like states formed in collisions of mixed-state He-like ions with $\mathrm{H}_{2}$ targets [57,59,62-64]. (6) The normalization to model calculations [37,75-78], where measured cross sections are normalized to theory, as for example, in the normalization of electron impact ionization measurements to convergent close coupling calculations [37].

Next, we shall discuss in more detail our results for He-like and Be-like ion beams produced in TANDEM accelerators in the context of our development of method 5 above.

\section{1. $1 s 2 s^{3} S$}

In Ref. [60], Schiebel et al. reported on the first determination of the $1 s 2 s^{3} S$ fraction in $\mathrm{Si}^{12+}$ ions. They measured the Kx-ray yield of the state at a certain distance from the production area and determined the fraction based on the theoretical value of the lifetime of the state. In their report they also introduced another technique involving the measurements of the K x-ray yields of Ar targets for incoming $\mathrm{Si}^{(11-13)+}$ ions. Their technique applies to nearly symmetric collisions, where K-shell to K-shell vacancy-transfer cross sections are large. Their results for both approaches agree within statistics showing the fraction to depend on both the thickness, as well as the atomic number of the foil. The fraction linearly increases with collision energy over the investigated range. In a similar study, Terasawa et al. used $\mathrm{F}^{7+}\left(1 s^{2}{ }^{1} S, 1 s 2 s^{1,3} S\right)$ mixed-state ion beams to determine the metastable fraction based on target $\mathrm{K}$ x-ray data obtained from the bombardment of the $\mathrm{F}^{7+}$ beams on thin Ti layers evaporated on carbon foils [61]. They showed that the metastable fraction follows a slow increase with collision energy reaching a plateau of $\sim 30 \%$ around an energy of $2 \mathrm{MeV} / \mathrm{u}$.

In Ref. [72], the fraction of metastable $\mathrm{He}\left(1 s 2 s^{3} S\right)$ produced by electron capture neutralization of slow 25-90 keV He${ }^{+}$ions in $\mathrm{H}_{2}$ gas was measured by photon-particle coincidence. The study showed that the fraction of metastable ions reached as high as 70\% at lower energies. In Ref. [37], the metastable fraction was determined in electron impact ionization experiments for $\mathrm{Li}^{+}$mixed-state 
ions. The data were compared to theoretical convergent close coupling calculations resulting in a $1 s 2 s^{3} S$ metastable fraction of $13 \%$. Similar studies were also reported for other ions as well [78]. Recently, the photoionization measurements for mixed-state $\mathrm{C}^{4+}$ ions were compared to dielectronic recombination cross sections of $\mathrm{C}^{5+}+e^{-}$through the principle of detailed balance [74]. The metastable fraction of $10.8 \%$ was thus determined in very good agreement with calculations based on relativistic many-body perturbation theory.

In Ref. [62] an alternative method was proposed based on the measurement of relative Auger electron yields from doubly excited $1 s 2 l 2 l^{\prime}$ states formed in collisions of $\mathrm{B}^{3+}$ mixed-state ions with $\mathrm{H}_{2}$. The method is based on the assumption that the $1 s 2 s 2 p^{4} P$ state is exclusively produced by capture to the $1 s 2 s^{3} S$ metastable state, while the $1 s 2 p^{2}{ }^{2} D$ is primarily produced from the $1 s^{2}{ }^{1} S$ by resonant transfer and excitation (RTE). The method does not suffer from experimental parameters uncertainties as it incorporates the ratio of the ${ }^{4} P$ and ${ }^{2} D$ peaks in the same spectrum. However, it uses theoretical calculations for the cross sections of RTE and electron capture, as well as modeling calculations for the corrected yield detection of the long-lived $1 s 2 s 2 p^{4} P$ state. It was shown that the metastable fraction produced using foil stripping remained practically constant near $25 \%$ over the incident energy range of $0.85-9 \mathrm{MeV}$. In comparison, the fraction produced using gas stripping showed a strong dependence on the incident beam energy reaching the maximum of $25 \%$ around the collision energy of $5 \mathrm{MeV}$. The method was also applied to low atomic number $Z_{p}$ elements with $4 \leq Z_{p} \leq 9$ [63].

In a similar approach, based on the same assumptions, Benis et al. [64] proposed a different method that used the ratio of the yields of the ${ }^{4} P$ and ${ }^{2} D$ lines in the same spectrum, but did not involve any theoretical cross sections of the ${ }^{4} P$ and ${ }^{2} D$ states or any model calculations for the solid angle correction due to the long decay of the ${ }^{4} P$ state. Actually, the critical assumptions are that the ${ }^{4} P$ and ${ }^{2} D$ lines result only from the $1 s 2 s^{3} S$ metastable and $1 s^{2}{ }^{1} S$ ground states, respectively, without any additional conditions about the particulars of the population processes involved. Instead, the technique requires two independent measurements of the same electron spectrum at the same collision energy, but using mixed beams having quite different $1 s 2 s^{3} S$ metastable beam fraction in each. Then, the metastable fraction is determined only by the normalized yields $Z$ of the ${ }^{4} P$ and ${ }^{2} D$ peaks as:

$$
f_{3 S}^{[i]}=Z^{[i]}\left({ }^{4} P\right) \frac{Z_{1}\left({ }^{2} D\right)-Z_{2}\left({ }^{2} D\right)}{Z_{1}\left({ }^{2} D\right) Z_{2}\left({ }^{4} P\right)-Z_{2}\left({ }^{2} D\right) Z_{1}\left({ }^{4} P\right)}, \quad i=1,2,
$$

where $i=1,2$ refers to the high and low metastable fractions, respectively. Thus, the method does not suffer from uncertainties arising either from theoretical calculations or experimental parameters. The only requirement is that the two spectra have appreciably different fractions. Typical spectra used in such fraction determinations are shown in Figure 2. The boron spectra were obtained after colliding the TANDEM delivered $\mathrm{B}^{2+}$ beam with thin carbon foils (foil post-stripping, FPS) or with Ar gas targets (gas post-stripping, GPS). The carbon spectra were obtained either with FPS of the $C^{3+}$ ions on thin carbon foils or stripping the incident $\mathrm{C}^{-}$beam inside the terminal with $\mathrm{N}_{2}$ gas targets (gas terminal stripping-GTS).

The obtained fractions are summarized in Table 2. It is seen that the fraction obtained for carbon is much smaller than that for boron. An explanation for this was proposed in Ref. [63] considering the K-vacancy sharing between the projectile and the target. Indeed, in the near-symmetric stripping process (i.e., $\mathrm{C}^{4+}$ traversing carbon foils) the K-vacancy transfer probability is close to $1 / 2$ [79]. In this case, the K-vacancy on an incident metastable $\mathrm{C}^{4+}\left(1 s 2 s^{3} S\right)$ ion will be transferred to the stripper carbon atom, leaving approximately half of the projectile ions in the ground state. For ions other than carbon, the more asymmetric K-vacancy transfer probability for other He-like beams is about an order of magnitude smaller and thus the reduction of the corresponding metastable fraction is negligible. 


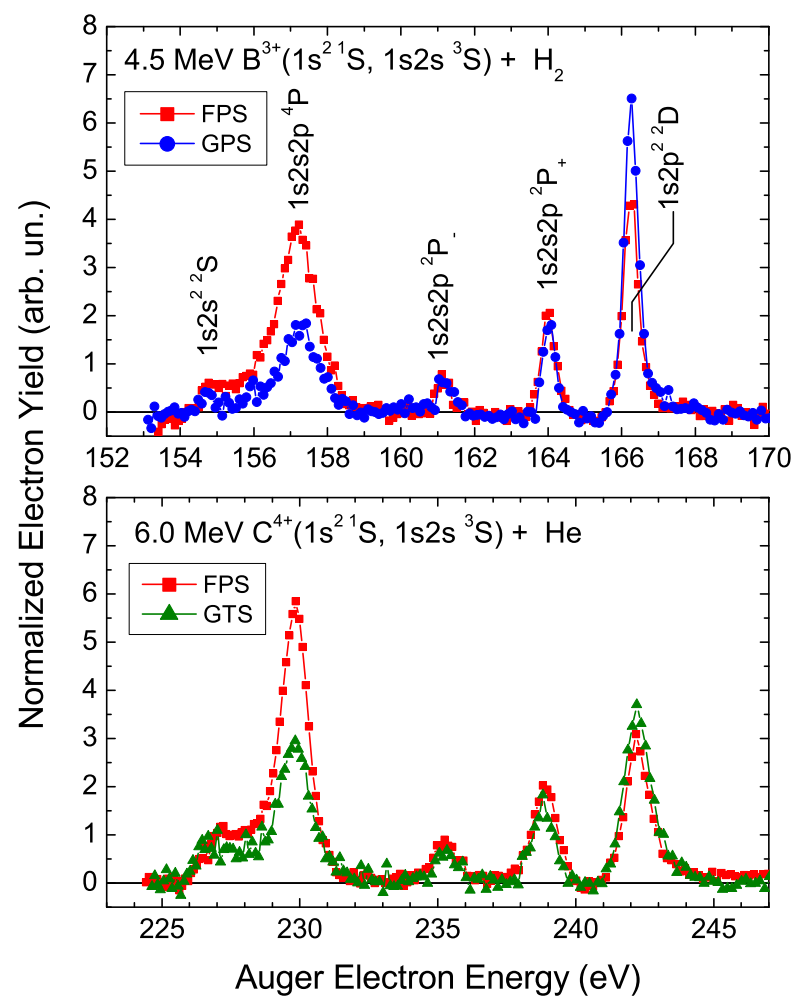

Figure 2. Auger KLL spectra obtained in collisions of $4.5 \mathrm{MeV} \mathrm{B}^{3+}$ with $\mathrm{H}_{2}$, as reported in Ref. [64], and of $6.0 \mathrm{MeV} \mathrm{C}^{4+}$ with He. The $\mathrm{B}^{3+}$ and $\mathrm{C}^{4+}$ beams were produced: [Red squares] After post-stripping the incident $\mathrm{B}^{2+}$ and $\mathrm{C}^{3+}$ beams in thin carbon foils (FPS). [Blue dots] After post-stripping the incident $\mathrm{B}^{2+}$ beam in Ar gas (GPS). [Green triangles] after stripping the incident $\mathrm{C}^{-}$ beam in the accelerator terminal in $\mathrm{N}_{2}$ gas (GTS). A smaller ratio of ${ }^{4} P$ to ${ }^{2} D$ yields implies a smaller metastable fraction.

Table 2. Results of the experimental determination (using Equation (1)) of the $1 s 2 s^{3} S$ metastable fraction $f_{3 S}$ on target. FPS: foil post-stripping, GPS: gas post-stripping, GTS: gas terminal stripping. The uncertainties of the fractions are given in the adjacent parentheses.

\begin{tabular}{ccccc}
\hline $\begin{array}{c}\text { Stripping } \\
\text { Method }\end{array}$ & $\begin{array}{c}\text { Incident } \\
\text { Ion }\end{array}$ & $\begin{array}{c}\text { Stripping Energy } \\
\mathbf{M e V}\end{array}$ & $\begin{array}{c}\text { Final Energy } \\
\mathbf{M e V}\end{array}$ & $\begin{array}{c}f_{3 S} \\
\mathbf{\%}\end{array}$ \\
\hline FPS & $\mathrm{B}^{2+}$ & 4.5 & 4.5 & $42(10)$ \\
GPS & $\mathrm{B}^{2+}$ & 4.5 & 4.5 & $18(5)$ \\
FPS & $\mathrm{C}^{3+}$ & 6.0 & 6.0 & $16(3)$ \\
GTS & $\mathrm{C}^{-}$ & 1.2 & 6.0 & $7(2)$ \\
\hline
\end{tabular}

We note that for GTS at low enough stripping energies, the metastable fraction can be quite small, almost negligible, compared to the fraction obtained by foil stripping. Such a situation is shown in Figure 3. This extreme condition resulting in the biggest differences between the fractions of each measurement will give the most accurate determination of the metastable fraction. In Section 5.1 we shall analyze the particular information offered by such spectra. As a final comment, the methods of Refs. $[62,64,80]$ are clearly much easier to apply since they involve running the same ion beam, while only varying the stripping conditions, rather than measuring the target $\mathrm{K}$ x-rays for $\mathrm{H}$-like, He-like and Li-like ions separately to obtain the fraction as used in Ref. [61]. 


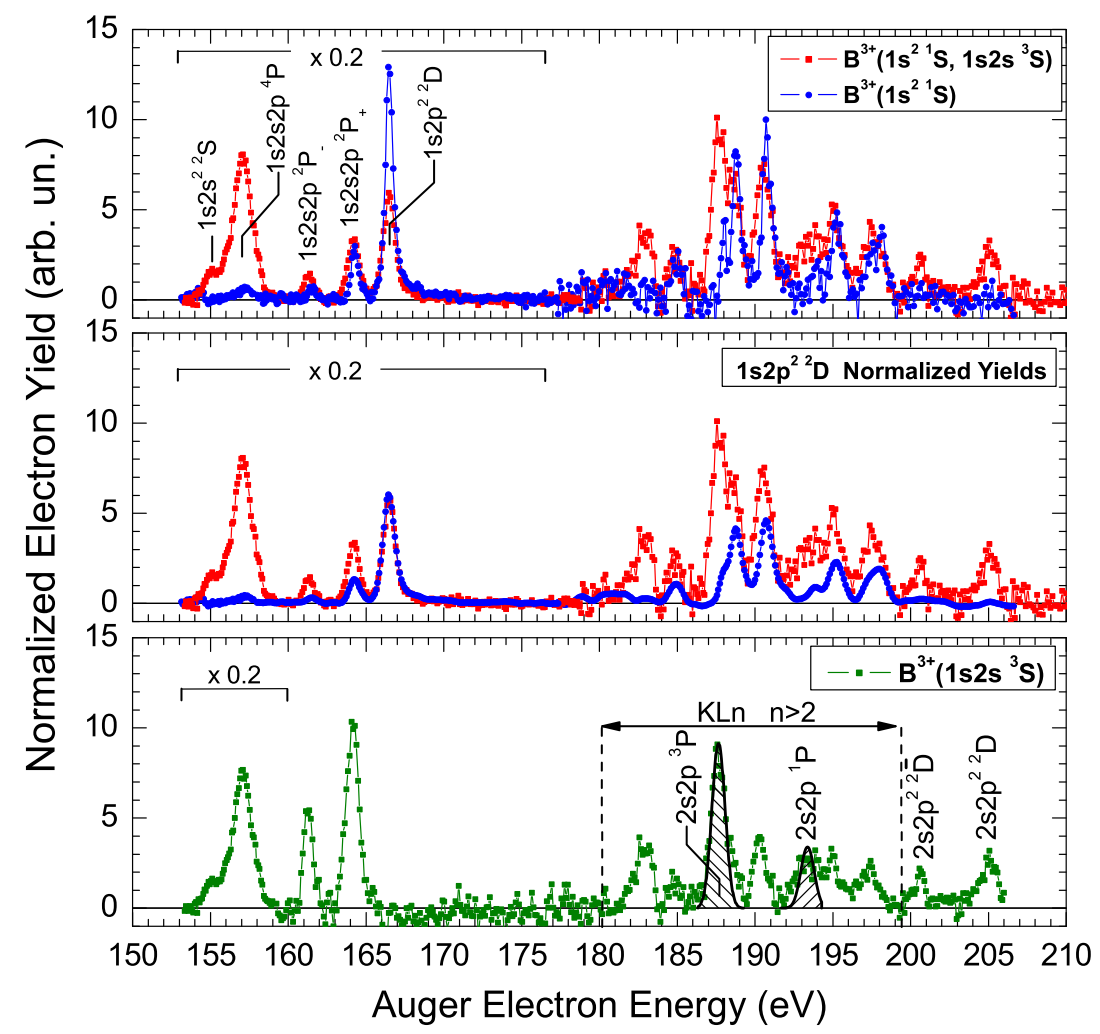

Figure 3. (Top) Li-like Auger spectra obtained in collisions of $4 \mathrm{MeV} \mathrm{B}^{3+}$ with $\mathrm{H}_{2}$ targets. The red squares correspond to the mixed-state $\left(1 s^{2}{ }^{1} S, 1 s 2 s^{3} S\right)$ beam, while the blue dots to the almost pure ground state $1 s^{2}{ }^{1} S$, as evident by the very small contribution of the ${ }^{4} P$ peak. The high fraction spectrum was obtained with FPS, while the low fraction with GTS. (Middle) Same as in the top graph, but here the ground state spectrum was convoluted with the slightly larger energy resolution of the mixed-state spectrum and then normalized to the $1 s 2 p^{2}{ }^{2} D$ line. (Bottom) Li-like Auger spectrum corresponding just to the $1 s 2 s^{3} S$ metastable state. The spectrum resulted from the subtraction of the two normalized spectra of the middle graph.

\section{2. $1 s^{2} 2 s 2 p^{3} P$}

Be-like ions produced in TANDEM accelerators are delivered in the ground $1 s^{2} 2 s^{2}{ }^{1} S$ and the metastable $1 s^{2} 2 s 2 p^{3} P_{J}$ states. The metastable lifetimes are in the $\mu$ s to s range depending on atomic number $Z_{p}$ and angular momentum $J$ [81-83]. During collisions with $\mathrm{H}_{2}$ targets, the needle ionization of the $1 s$ electron [84] of the $1 s^{2} 2 s 2 p^{3} P$ state results in the production of the $1 s 2 s 2 p$ configuration. In the LS coupling scheme the $2 s$ and $2 p$ electrons interact strongly as parts of the same shell and are negligibly affected by the K-shell configuration. Thus, even after the $1 s$ ionization, the L-shell electrons should maintain their ${ }^{3} P$ coupling. In this spirit, the only viable states are the $1 s 2 s 2 p{ }^{4} P$ and the $1 s\left(2 s 2 p^{3} P\right)^{2} P$. This is very similar to what also occurs in photo-ionization of Be-like ions [83,85-88]. Similarly, $1 s$ ionization of the $1 s^{2} 2 s^{2}{ }^{1} S$ ground state results in the Li-like $1 s 2 s^{2}{ }^{2} S$ intermediate state. Since the 1 s needle ionization process is not expected to depend strongly on the L-shell configuration, the K-vacancy production cross sections from the ground state and the metastable state can be expected to be equal, i.e., $\sigma_{1 s}\left(1 s^{2} 2 s^{2}\right)=\sigma_{1 s}\left(1 s^{2} 2 s 2 p^{3} P\right)$ as also assumed by Lee et al. [17]. In addition, the production population statistics of the ${ }^{4} P$ and ${ }^{2} P_{-}$states should result in the ratios $\sigma\left({ }^{4} P\right): \sigma\left({ }^{2} P_{-}\right)=2: 1$, as is obvious from the multiplicity of the states. Consequently, the following ratios of the production cross sections should be valid, i.e., $\sigma\left({ }^{2} S\right): \sigma\left({ }^{4} P\right): \sigma\left({ }^{2} P_{-}\right)=3: 2: 1$. Then the metastable fraction $f_{3 P}$ is obtained as [89]:

$$
f_{3 P} \equiv\left[1+\frac{Z\left({ }^{2} S\right)}{Z\left({ }^{4} P\right)+Z\left({ }^{2} P_{-}\right)}\right]^{-1}=\left[1+\frac{1}{3} \frac{Z\left({ }^{2} S\right)}{Z\left({ }^{2} P_{-}\right)}\right]^{-1},
$$


where $Z$ denotes the normalized electron yields of the corresponding state in the Auger spectrum.

In Figure 4, we reproduce high resolution electron spectra obtained in collisions of $17.5 \mathrm{MeV} \mathrm{O}^{4+}$ and 6.6 $\mathrm{MeV} \mathrm{C}^{2+}$ with $\mathrm{H}_{2}$ targets, initially reported in Ref. [89]. The measurements were performed with our ZAPS apparatus located at the tandem accelerator facility of "Demokritos". As can be seen, the ${ }^{4} P$ peak has an asymmetry towards the lower energy wing. This is due to the metastability of the state that results in its decay all the way from the gas cell to the entry of the spectrometer. This feature strongly affects the detection solid angle as compared to a prompt state that decays inside the gas cell. We have studied in detail this behavior and results have been reported in the literature $[90,91]$. In Figure 4, the reproduction of the asymmetry in Monte Carlo type simulations, using the ion-optics package SIMION 8.1 [92], is presented. Moreover, the small asymmetry in the peak near $425 \mathrm{eV}$, evident in the oxygen spectrum, is due to the additional low-intensity $1 s 2 s^{2} 2 p^{3} P$ Auger line. This state can be formed from the $1 s^{2} 2 s^{2}{ }^{1} S$ ground state via $1 s \rightarrow 2 p$ excitation and decays promptly to the $1 s^{2} 2 p$ final state [17]. The line spectra were fitted with constant width Voigt profiles, except for the ${ }^{4} P$ peak, and the metastable fractions were determined. In the case of carbon, a contribution of $17 \%$ for the $1 s 2 s^{2} 2 p^{3} P$ line was assumed, as obtained in the oxygen case. Thus, the metastable fraction values of $70 \pm 5 \%$ and $67 \pm 5 \%$ were obtained, for the case of carbon and oxygen, respectively. It is worth mentioning that these large fractions for the metastable $1 s^{2} 2 s 2 p^{3} P$ beam component, typically larger than the ground state component for these low $Z_{p}$ ions, clearly facilitates studies involving this state.

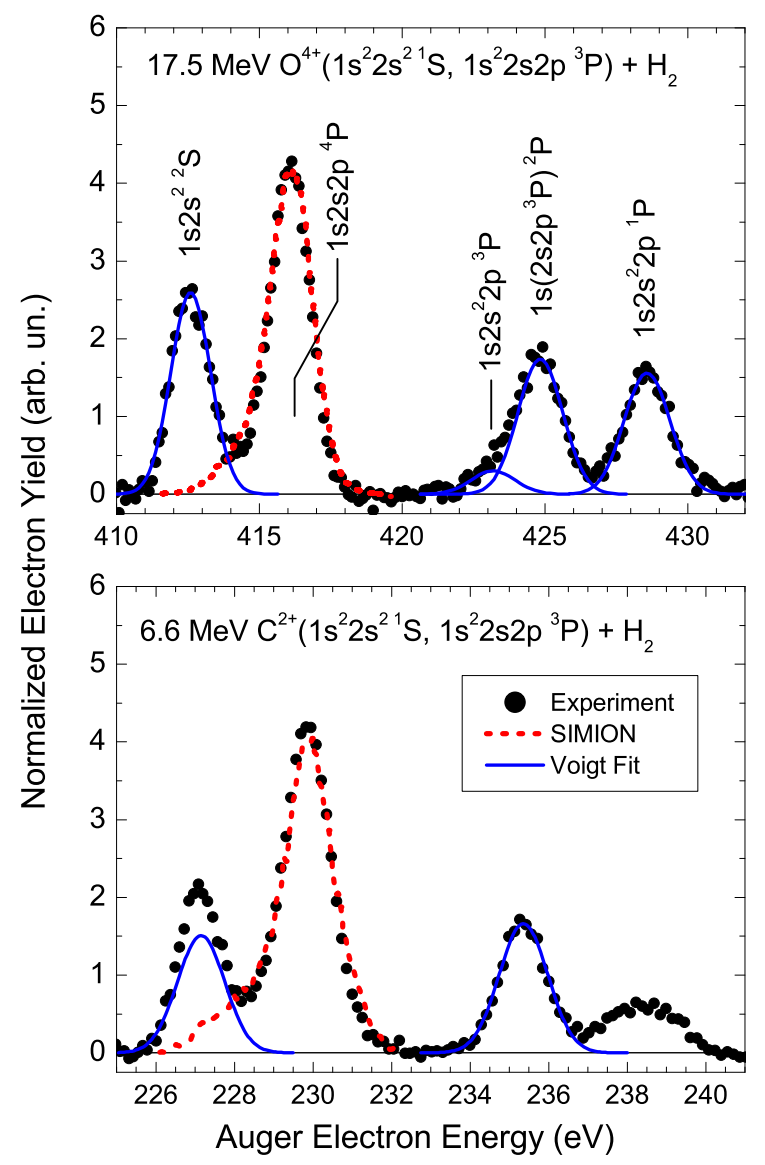

Figure 4. Auger KLL spectra obtained in collisions of $17.5 \mathrm{MeV} \mathrm{O}^{4+}$ and $6.6 \mathrm{MeV} \mathrm{C}^{2+}$ with $\mathrm{H}_{2}$ targets (from Ref. [89]). SIMION simulations of the ${ }^{4} P$ line distributions are shown by the short dash line in excellent agreement with the measurements. The solid lines correspond to Voigt profile least square fits of the Auger lines. 


\section{Case Studies: Results and Discussion}

\subsection{Doubly and Triply Excited Li-Like States}

In Figure 3 (top), we present measurements of the complete Li-like Auger spectrum obtained in collisions of $4 \mathrm{MeV} \mathrm{B}^{3+}$ with $\mathrm{H}_{2}$ targets. As shown, the same electron spectrum was obtained in two independent measurements at the same collision energy, but using mixed-state beams having quite different $1 s 2 s^{3} S$ metastable fractions. The high fraction spectrum was obtained with FPS, while the low fraction with GTS, in order to maximize their difference. Judging by the very small contribution of the ${ }^{4} P$ peak in the low fraction spectrum, it can safely be considered negligible compared to the high value fraction. Thus, the two measurements correspond to the spectra of $\mathrm{B}^{3+}\left(1 s^{2}{ }^{1} S, 1 s 2 s^{3} S\right)$ mixed and $B^{3+}\left(1 s^{2}{ }^{1} S\right)$ pure ground states, respectively. Such fraction-controlled measurements provide the condition for a clear separation of the contribution of the metastable component. The only necessary requirement is to normalize the spectrum of the ground state to that of the mixed-state with respect to a peak that is unambiguously formed by the ground state alone. In our case, this peak is the $1 s 2 p^{2}{ }^{2} \mathrm{D}$ which is primarily populated by the ground state through the process of transfer and excitation. Then, the Auger spectrum of the pure $1 s 2 s^{3} S$ metastable state is obtained by a simple subtraction of the two spectra. Application of this approach can be found in Ref. [58]. We should mention though that the applicability of the method also highly relies on the efficient detection of the long-lived $1 s 2 s 2 p^{4} P$ state, particularly in the low fraction case. Poor efficiency due to the geometry of the experiment may erroneously give the impression of an almost pure ground state beam, while in fact most of the ${ }^{4} P$ is just not detected, as it might mostly decay after the analyzer [90]. For our ZAPS setup, with its efficient two-dimensional position sensitive detector and the judicious positioning of the spectrograph with respect to the target, the detection efficiency is large enough to avoid such difficulties.

Here, we followed this method for the spectra shown in Figure 3 (top). In more detail, due to the different energy resolution of the two spectra, due to beam straggling for the FPS mixed-state spectrum, we first convoluted the ground state spectrum with the slightly larger energy resolution width of the mixed-state spectrum, and then normalized the two spectra with respect to the $1 s 2 p^{2}{ }^{2} \mathrm{D}$ peak, as shown in Figure 3 (middle). Finally, after subtracting the two normalized spectra, the resulting spectrum corresponding to the $1 s 2 s^{3} S$ metastable state is obtained. The result is shown in Figure 3 (bottom).

The first important result of this approach is the direct separation of the two beam component contributions. The $1 s 2 s^{3} S$ contributions are seen to be absent in the pure ground state spectrum and can be identified even by the naked eye when examining the two spectra in comparison. Indeed, aside from the long-lived $1 s 2 s 2 p^{4} P$ state, the triply excited $2 s 2 p^{2}{ }^{2} D$ state is clearly evident in the energy region between 200 and $207 \mathrm{eV}$. This state is populated predominantly by RTE and it Auger decays either back to the $1 s 2 s^{3} S$ state or to the $1 s 2 s{ }^{1} S$ and $1 s 2 p^{3} P$ states (these last two are separated by less then $20 \mathrm{meV}$ [34] and cannot be resolved), thus resulting in the two observed lines noted as $2 s 2 p^{2}{ }^{2} D$ and $2 s 2 p^{2}{ }^{2} \bar{D}$, respectively. These triply excited states, cannot be straightforwardly populated by photo-ionization, thus they were studied by our group in ion-atom collisions for isoelectronic projectiles with atomic number $5 \leq Z_{p} \leq 9$. This investigation also resulted in some of the first tests of R-matrix calculations for open shells, eventually bringing them into good agreement $[34,93,94]$. Another clearly separated Auger line is the He-like hollow state $2 s 2 p^{3} P$, the formation of which is discussed in Section 5.2 below.

The second and possibly even more important result is the separation of the contributions of states that can be populated from both ground and metastable components. These states appear in both the ground and the mixed-state spectra preventing a straightforward determination of their production cross sections in a single measurement involving only mixed-state beams. However, in our dual measurement approach, the two contributions can be separated and production cross sections for the $1 s 2 s^{3} S$ metastable state and the $1 s^{2}{ }^{1} S$ ground state, can be safely obtained. In our spectra shown in Figure 3, these states include the Auger KLL lines $1 s 2 s^{2}{ }^{2} S, 1 s 2 s 2 p^{2} P_{+}$and $1 s 2 s 2 p^{2} P_{-}$states, as well as all the higher-lying $K L n$ states with $\mathrm{n} \geq 3$ mostly of the type $1 s 2 s\left({ }^{3} S\right) n l^{2} L$. 
Moreover, such studies can also provide important information about secondary processes. For example, a still open issue is the significance of the process of the selective cascade feeding of the $1 s 2 s 2 p^{4} P$ state from higher lying $1 s 2 s 2 l^{4} L$ quartet states populated by single electron capture in collisions of mixed-state He-like ions with gas targets. It is argued that since the population of $1 s 2 s n l^{2} L(n>2)$ doublet states is evident in the pure $1 s 2 s{ }^{3} S$ spectrum of Figure 2, the population of the corresponding $1 s 2 s n l{ }^{4} L(n>2)$ quartet states should also be considered a strong possibility. However, these quartet states cannot be seen [25] in the above Auger spectra since they have very small Auger rates and therefore preferentially radiatively decay to lower-lying quartet states via much stronger E1 transitions [95], ending on the $1 s 2 s 2 l^{4} P$ state, thus enhancing its yield. Our group is currently actively investigating, both experimentally and theoretically, these processes $[59,80,96]$. In addition, we have also developed a new version of the two measurement technique in which we can now separate the contributions of the ground and metastable components, even when no pure ground state is available [59]. This new technique, similarly involves two independent measurements at the same collision energy using mixed beams with different $1 s 2 s^{3} S$ metastable fractions. However, subtraction of the two spectra is not viable, but rather extraction of the single differential production cross section of the Li-like doubly excited states is obtained for both the ground and metastable components simultaneously. Details are given in Ref. [59].

\subsection{Doubly Excited He-Like States}

In Figure 5 (top), we present measurements of the complete Li-like Auger spectrum obtained in collisions of $15 \mathrm{MeV} \mathrm{C}^{4+}$ with He targets. The conditions are similar to the data shown in Figure 3, i.e., the electron spectra were obtained in two independent measurements at the same collision energy, but using mixed beams having different $1 s 2 s^{3} S$ metastable fractions. The high fraction spectrum was obtained after stripping the incident $\mathrm{C}^{-}$beam inside the terminal with thin carbon foils (foil terminal stripping - FTS), while the low fraction with GTS. We chose this collision energy as the He-like doubly excited states $2 s 2 p^{1,3} \mathrm{P}$, of interest here, are also quite pronounced. However, at these high collision energies the delivered beams result only in mixed-state beams, thus preventing the use of a pure ground state as done in the previous section. The accompanied Auger KLL spectra show the expected behavior, i.e., that the yields for the ${ }^{2} S,{ }^{4} P,{ }^{2} P_{-}$and ${ }^{2} P_{+}$states, arising primarily from the metastable component are reduced for the low fraction condition, as opposed to that for the ${ }^{2} D$ state, arising mostly from the ground state, where the yield is increased.

The above fraction-controlled measurements provide valuable information about the processes involved in the production of the doubly excited $2 s 2 p^{1,3} P$ states. Indeed, as can be seen in Figure 5 (top), the relatively small reduction of the metastable percentage results in a large reduction in the yield of the $2 s 2 p^{3} P$ state, while the yield of the accompanied $2 s 2 p^{1} P$ state remains almost unaffected. This behavior implies that the $2 s 2 p^{3} P$ state is populated primarily by the $1 s 2 s^{3} S$ metastable state. Indeed, the $2 s 2 p^{3} P$ state can be straightforwardly formed by $1 s \rightarrow 2 p$ single electron excitation, a process of large cross section due to its dipole character. On the other hand formation from the $1 s^{2}{ }^{1} S$ ground state would require higher order processes such as $1 s \rightarrow 2 p$ excitation with spin flip and transfer loss ( $2 p$ transfer, $1 \mathrm{~s}$ loss) which are much less probable at these collision energies compared to the direct $1 s \rightarrow 2 p$ excitation.

At this point we should also consider possible contributions from the $1 s 2 s{ }^{1} S$ state, so far not discussed and assumed to be negligible. Even though its fraction is in general very small $(<5 \%)$ and does not seem to appreciably contribute to the doubly excited KLL states, its contribution to the $2 s 2 p^{1,3} P$ states should not be neglected. Indeed, the $2 s 2 p^{3} P$ could be populated from the $1 s 2 s{ }^{1} S$ state by direct $1 s \rightarrow 2 p$ excitation with spin flip, reducing significantly its cross section. Therefore, based on the data of Figure 5 (top), we may safely state that the $2 s 2 p^{3} p$ state is primarily formed from the $1 s 2 s^{3} S$ state by $1 s \rightarrow 2 p$ single electron excitation. Alternatively, the situation for the $2 s 2 p^{1} P$ state is more complicated. The very small decrease of its Auger yield following the reduction of the metastable part of the beam implies that the $1 s 2 s^{3} S$ state has just a small contribution. Indeed, $2 s 2 p^{1} P$ 
can be populated from the $1 s 2 s^{1} S$ state by direct $1 s \rightarrow 2 p$ excitation, but it may also be populated from the $1 s^{2}{ }^{1} S$ ground state via the second order processes of double excitation $(1 s \rightarrow 2 s, 1 s \rightarrow 2 p)$ and/or transfer loss ( $2 p$ transfer, $1 s$ loss). Transfer loss is also viable in the production of the $1 s 2 s^{3} S$ state. These second order processes, although of smaller cross section compared to the direct $1 s \rightarrow 2 p$ excitation, have an increased weight due to the much higher ground state fractions they can arise from. Therefore, the formation of the $2 s 2 p^{1} P$ state can most likely be attributed largely to the $1 s \rightarrow 2 p$ excitation of the $1 s 2 s{ }^{1} S$ state and to a lesser extent to higher order processes involving the $1 s^{2}{ }^{1} S$ ground and $1 s 2 s^{3} S$ metastable states. Atomic orbital close coupling (AOCC) calculations in progress confirm these observations.

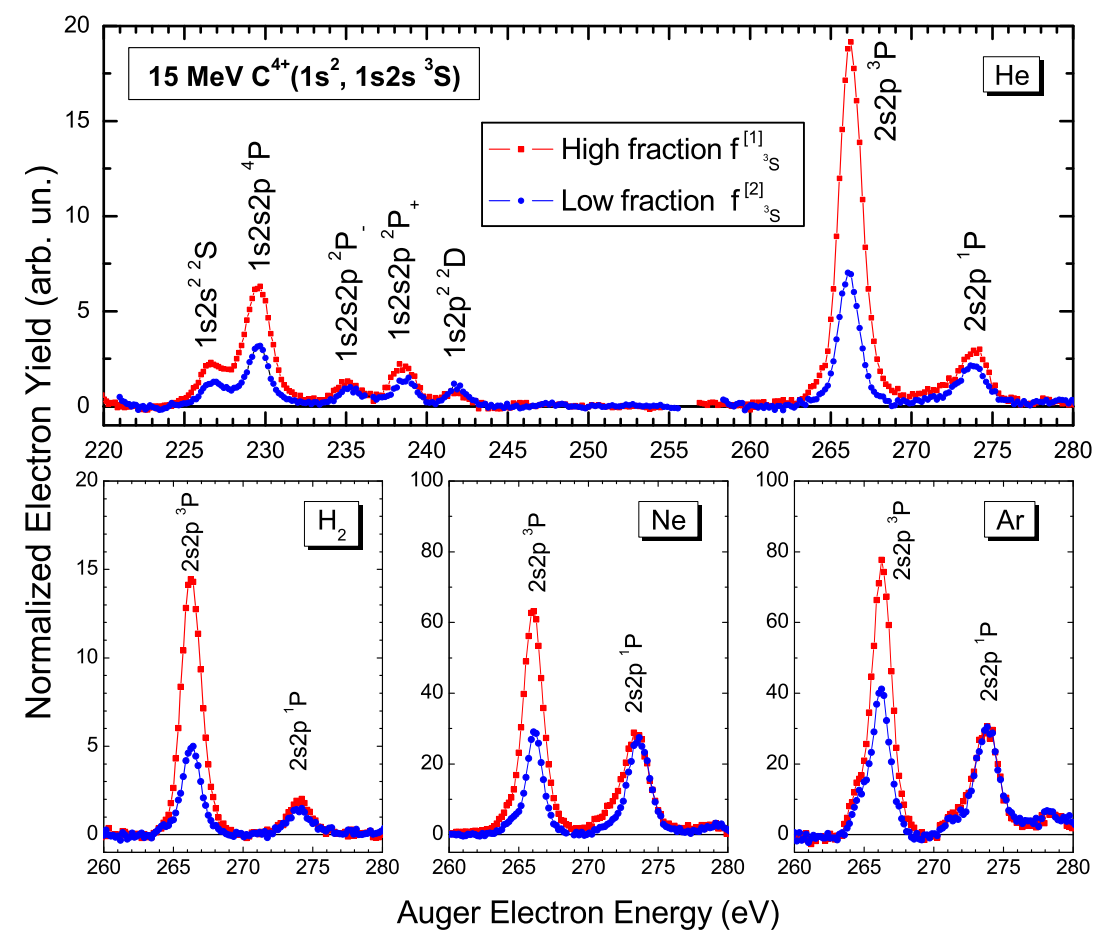

Figure 5. (Top) Li-like Auger spectra obtained in collisions of $15 \mathrm{MeV} \mathrm{C}^{4+}$ with He targets. The red squares correspond to the mixed-state beam with higher value for the $1 s 2 s^{3} S$ metastable fraction, while the blue dots to the lower value. The high fraction spectrum was obtained with FTS, while the low fraction with GTS. (Bottom) The He-like doubly excited $2 s 2 p^{1,3} \mathrm{P}$ states obtained in collisions with $\mathrm{H}_{2}$, $\mathrm{Ne}$ and Ar gas targets.

In Figure 5 (bottom), we also present measurements of the $2 s 2 p^{1,3} \mathrm{P}$ states in collisions of $15 \mathrm{MeV}$ $\mathrm{C}^{4+}$ with $\mathrm{H}_{2}, \mathrm{Ne}$ and Ar targets. The $\mathrm{H}_{2}$ data are quite similar to the He case, as expected for simple targets, and are interpreted accordingly. For the case of the more complicated targets of $\mathrm{Ne}$ and Ar, it is seen that the main population channel for the $2 s 2 p^{3} P$ state is still the $1 s 2 s^{3} S$ state, as evident by the large change in its yield following the relatively small reduction of the metastable beam percentage. The yield of the $2 s 2 p^{1} P$ state though seems essentially unaffected, implying that the second order processes involving the $1 s^{2}{ }^{1} S$ ground and $1 s 2 s{ }^{3} S$ metastable states are now as significant as the $1 s \rightarrow 2 p$ excitation from the $1 s 2 s^{1} S$ state.

\section{Summary}

In this work we examined the use of naturally occurring mixed-state ionic beams in atomic collision investigations. Our study primarily focused on the He-like $1 s 2 s^{1,3} S$ and Be-like $1 s^{2} 2 s 2 p^{3} P$ metastable states, typically delivered by TANDEM accelerators in fractions as large as $30 \%$ and $70 \%$, respectively. These metastable states, particularly for low atomic number $Z_{p}$ ions, are extremely long-lived and normally are delivered to the target without significant loss. Zero-degree Auger 
projectile spectroscopy was used to obtain state-selective information about the excitation state of the projectile ions after collisions with gas targets. The metastable beam fractions can be varied since they depend on stripper density, stripper medium and stripping ion energy. This dependence has led to a technique for the determination of the metastable fraction and separation of each component's contribution to the spectrum. The production of mixed-state He-like and Be-like ionic beams, the lifetimes of their metastable components and the techniques used to determine their fractional composition were reviewed. Our group has developed such a technique involving two different measurements under the same conditions, but with different metastable fractions. Corresponding results from older, as well as new measurements, were presented in detail.

Accordingly, we reported on studies involving the production of $\mathrm{He}$ - and Li-like states obtained in collisions of $4 \mathrm{MeV} \mathrm{B}^{3+}$ with $\mathrm{H}_{2}$ targets. Using mixed and pure ground state beams at the same collision energy, we showed how to separate the Auger spectrum contributions from just the metastable beam component. In this way, an Auger electron spectrum corresponding to a pure $1 s 2 s^{3} S$ metastable beam is obtained, where the formation of $1 s 2 \ln l^{\prime 2} L K L n$, as well as the $2 s 2 p^{1,3} P$ and $2 s 2 p^{2}{ }^{2} D$ doubly- and triply-excited states, respectively, are observed and discussed accordingly. In a different application, using a $15 \mathrm{MeV} \mathrm{C}^{4+}$ mixed-state ion beam in collisions with $\mathrm{H}_{2}, \mathrm{He}, \mathrm{Ne}$ and Ar targets, the specific role of each of the mixed-state beam components is evaluated. The various processes contributing to the formation of the $2 s 2 p^{1,3} P$ are then much more clearly identified, facilitating the comparison to theoretical calculations.

To date, while it is not difficult to know whether your ion beam contains metastable states, or even measure the amount of metastable fraction using one of the methods described here, it has not yet become feasible to "dial in" the exact amount of metastable fraction you would like to use in your experiment. However, this is in principle possible once the ion source conditions or the charge-stripping conditions affecting the metastable fraction have been accurately calibrated. In a TANDEM, where the exact amount of $1 s 2 s^{3} S$ fraction relies on the density of the stripper medium and the stripping energy this should be possible with the careful control of the stripper density, either foil thickness or gas pressure. In the upcoming upgrade of the "Demokritos" tandem Van de Graaff accelerator, a new ion source and an accurate pressure control system to set and monitor the terminal gas stripper pressure should allow such a calibration. Knowing accurately the specific amount of metastable content without having to measure it each time would clearly be very useful. While existing computer codes can accurately predict the ionic charge distributions following stripping $[47,50,97]$, to our knowledge there are no codes to also explicitly predict the expected amount of metastable fractions. Clearly, this would also be a useful development. Ongoing theoretical developments on the use of three $[98,99]$ and possibly even four active electrons in AOCC calculations of transfer and excitation processes in collisions of He-like ions in the $1 s 2 s^{1,3} S$ state with $\mathrm{He}$ and $\mathrm{H}$ targets are presently under way and should soon provide new and interesting results.

Author Contributions: E.P.B. and T.J.M.Z. conceived, designed, participated in the experiments, the data analysis and prepared the manuscript; I.M., A.L. and S.N. participated in the experiments and the data analysis.

Funding: During the period (2012-2015) we acknowledge support by the project APAPES co-financed by the European Union (European Social Fund-ESF) and Greek national funds through the Operational Program "Education and Lifelong Learning" of the National Strategic Reference Framework (NSRF) Research Funding Program: THALES. Investing in knowledge society through the European Social Fund, grant number MIS 377289. For the period of (2017-2021), we acknowledge support by the project "CALIBRA/EYIE" (MIS 5002799) which is implemented under the Action "Reinforcement of the Research and Innovation Infrastructures", funded by the Operational Programme "Competitiveness, Entrepreneurship and Innovation" (NSRF 2014-2020) and co-financed by Greece and the European Union (European Regional Development Fund).

Acknowledgments: We would like to thank David Manura of Scientific Instrument Services for help with Lua programming of SIMION. We would like to express our gratitude to the former director of INPP, Sotirios Harissopulos, as well as to Dr Anastasios Lagoyannis for their support to the APAPES project. Last, but not least, we would like to thank both the KSU and Demokritos TANDEM accelerator staff for their invaluable help during beamtimes.

Conflicts of Interest: The authors declare no conflict of interest. 


\section{Abbreviations}

The following abbreviations are used in this manuscript:

$\begin{array}{ll}\text { APAPES } & \text { Atomic Physics with Accelerators: Projectile Electron Spectroscopy } \\ \text { AOCC } & \text { Atomic Orbital Close Coupling } \\ \text { ZAPS } & \text { Zero-degree Auger Projectile Spectroscopy } \\ \text { TANDEM } & \text { The two-stage (tandem) Van de Graaff accelerator } \\ \text { FPS } & \text { Foil Post-Stripping } \\ \text { FTS } & \text { Foil Terminal Stripping } \\ \text { GPS } & \text { Gas Post-Stripping } \\ \text { GTS } & \text { Gas Terminal Stripping } \\ \text { RTE } & \text { Resonant Transfer and Excitation }\end{array}$

\section{References}

1. Beyer, H.F.; Shevelko, P. Introduction to the Physics of Highly Charged Ions; Series in Atomic and Molecular Physics; Institute of Physics Publishing: Bristol, UK; Philadelphia, PA, USA, 2003.

2. Currell, F.J. The Physics of Multiply and Highly Charged Ions. Volume 1. Sources, Applications and Fundamental Processes; Series in Atomic and Molecular Physics; Springer-Science+Business Media: Dordrecht, The Netherlands, 2003.

3. Janev, R.K. Atomic and Molecular Processes in Fusion Edge Plasmas; Springer Science+Business Media, LLC: New York, NY, USA, 1995.

4. Stolterfoht, N.; Dubois, R.D.; Rivarola, R.D. Electron Emission in Heavy Ion-Atom Collisions; Springer Series on Atoms and Plasmas; Springer: Berlin, Germany, 1997.

5. Itikawa, Y. Molecular Processes in Plasmas; Springer: Berlin/Heidelberg, Germany; New York, NY, USA, 2007.

6. Müller, A. Electron-ion collisions: Fundamental processes in the focus of applied research. Adv. At. Mol. Opt. Phys. 2008, 55, 293-417.

7. V. Shevelko, H.T. Atomic Processes in Basic and Applied Physics; Springer: Berlin/Heidelberg, Germany; New York, NY, USA, 2012.

8. Janev, R.K.; Winter, H. State-selective electron capture in atom-highly charged ion collisions. Phys. Rep. 1985, 117, 265-387. [CrossRef]

9. Summers, H.P.; Dickson, W.J. Applications of Recombination. In Recombination of Atomic Ions; Graham, W.G.; Fritsch, W.; Hahn, Y.; Tanis, J., Eds.; NATO Advanced Study Institute Series B: Physics; Plenum Publishing Corporation: New York, NY, USA, 1992; Volume 296, pp. 31-48.

10. Kunze, H.J. Introduction to Plasma Spectroscopy, Springer Series on Atomic, Optical, and Plasma Physics; Springer: Berlin, Germany, 2009.

11. Tallents, G.J. An Introduction to the Atomic and Radiation Physics of Plasmas; Cambridge University Press: Cambridge, UK, 2018.

12. Becker, R.L.; Ford, A.L.; Reading, J.F. Multiple-vacancy production in the independent-Fermi-particle model. Phys. Rev. A 1984, 29, 3111-3121. [CrossRef]

13. Stolterfoht, N. Electron Correlation Processes in Energetic Ion-Atom Collisions. In Spectroscopy and Collisions of Few-Electron Ions; Ivascu, M., Florescu, V., Zoran, V., Eds.; World Scientific: Singapore; London, UK, 1989; p. 342.

14. Stolterfoht, N. Dynamics of Electron Correlation Processes in Atoms and Atomic Collisions. Phys. Scr. 1990, 42, 192-204. [CrossRef]

15. Mcguire, J. Multiple-Electron Excitation, Ionization, and Transfer in High-Velocity Atomic and Molecular Collisions. Adv. At. Mol. Opt. Phys. 1991, 29, 217-323.

16. Zouros, T.J.M. Resonant Transfer and Excitation Associated with Auger Electron Emission. In Recombination of Atomic Ions; Graham, W.G., Fritsch, W., Hahn, Y., Tanis, J., Eds.; NATO Advanced Study Institute Series B: Physics; Plenum Publishing Corporation: New York, NY, USA, 1992; Volume 296, pp. 271-300.

17. Lee, D.H.; Zouros, T.J.M.; Sanders, J.M.; Richard, P.; Anthony, J.M.; Wang, Y.D.; McGuire, J.H. K-shell Ionization of $\mathrm{O}^{4+}$ and $\mathrm{C}^{2+}$ ions in fast collisions with $\mathrm{H}_{2}$ and He targets. Phys. Rev. A 1992, 46, 1374-1387. [CrossRef] [PubMed] 
18. Montenegro, E.C.; Meyerhof, W.E.; McGuire, J.H. Role of two-center electron-electron interaction in projectile electron excitation and loss. Adv. At. Mol. Opt. Phys. 1994, 34, 249-300.

19. Zouros, T.J.M. Excitation and ionization in fast ion-atom collisions due to projectile electron-target electron interactions. In Applications of Particle and Laser Beams in Materials Technology; Misailides, P., Ed.; NATO Advanced Study Institute Series E: Applied Sciences; Kluwer Academic Publishers: Dordrecht, The Netherlands, 1995; Volume 283, pp. 37-52.

20. Zouros, T.J.M. Projectile-Electron-Target-Electron Interactions: Exposing the Dynamic Role of Electrons in Fast Ion-Atom Collisions. Comments At. Mol. Phys. 1996, 32, 291-313.

21. Zouros, T.J.M.; Benis, E.P.; Gorczyca, T.W. Large-angle elastic resonant and non-resonant scattering of electrons from $\mathrm{B}^{3+}\left(1 s^{2}\right)$ and $\mathrm{B}^{4+}(1 s)$ ions: Comparison of experiment and theory. Phys. Rev. A 2003, 68, R010701. [CrossRef]

22. Benis, E.P.; Zouros, T.J.M.; Gorczyca, T.W.; González, A.D.; Richard, P. Elastic resonant and non-resonant differential scattering of quasi-free electrons from $B^{4+}(1 \mathrm{~s})$ and $B^{3+}\left(1 \mathrm{~s}^{2}\right)$ ions. Phys. Rev. A 2004, 69, 052718.

23. Stolterfoht, N. High resolution Auger spectroscopy in energetic ion atom collisions. Phys. Rep. 1987, 146, 315-424. [CrossRef]

24. Zouros, T.J.M.; Lee, D.H. Zero Degree Auger Electron Spectroscopy of Projectile Ions. In Accelerator-Based Atomic Physics Techniques and Applications; Shafroth, S.M., Austin, J.C., Eds.; American Institute of Physics: Woodbury, NY, USA, 1997; Chapter 13, pp. 426-479.

25. Mack, M.; Niehaus, A. Radiative and Auger decay channels in K-Shell excited Li-like ions $(Z=6-8)$. Nucl. Instrum. Methods Phys. Res. B 1987, 23, 109-115. [CrossRef]

26. Lee, D.H.; Richard, P.; Sanders, J.M.; Zouros, T.J.M.; Shinpaugh, J.L.; Varghese, S.L. Electron Capture and Excitation Studied by State-Resolved KLL Auger Measurement in 0.25-2 MeV/u $F^{7+}\left(1 s^{2}{ }^{1} S, 1 s 2 s^{3} S\right)+$ $\mathrm{H}_{2}$ /He Collisions. Nucl. Instrum. Methods Phys. Res. B 1991, 56/57, 99-103. [CrossRef]

27. Tanis, J.A.; Landers, A.L.; Pole, D.J.; Alnaser, A.S.; Hossain, S.; Kirchner, T. Evidence for Pauli Exchange Leading to Excited-State Enhancement in Electron Transfer. Phys. Rev. Lett. 2004, 92, 133201. [CrossRef] [PubMed]

28. Mack, M.; Niehaus, A. Double electron capture by He-like ions: Collision energy dependence of the reaction window. Nucl. Instrum. Methods Phys. Res. B 1987, 23, 116-119. [CrossRef]

29. Zouros, T.J.M.; Lee, D.H.; Richard, P. Projectile $1 s \rightarrow 2 p$ Excitation Due to Electron-Electron Interaction in Collisions of $\mathrm{F}^{6+}$ and $\mathrm{O}^{5+}$ Ions with $\mathrm{He}$ and $\mathrm{H}_{2}$ Targets. Phys. Rev. Lett. 1989, 62, 2261-2264. [CrossRef] [PubMed]

30. Zouros, T.J.M.; Lee, D.H.; Sanders, J.M.; Shinpaugh, J.L.; Tipping, T.N.; Varghese, S.L.; Richard, P. High Resolution Studies of Electron Capture and Excitation by $0^{\circ}$ Projectile Electron Spectroscopy. Nucl. Instrum. Methods Phys. Res. B 1989, 40/41, 17. [CrossRef]

31. Zouros, T.J.M.; Lee, D.H.; Richard, P.; Sanders, J.M.; Shinpaugh, J.L.; Varghese, S.L.; Karim, K.R.; Bhalla, C.P. State-Selective Observation of Resonance Transfer-Excitation (RTE) in Collisions of $\mathrm{F}^{6+}$ with $\mathrm{He}$ and $\mathrm{H}_{2}$ Targets. Phys. Rev. A 1989, 40, 6246. [CrossRef]

32. Graham, W.G.; Fritsch, W.; Hahn, Y.; Tanis, J. (Eds.) Recombination of Atomic Ions; NATO Advanced Study Institute Series B: Physics; Plenum Publishing Corporation: New York, NY, USA, 1992; Volume 296.

33. Lee, D.H.; Richard, P.; Sanders, J.M.; Zouros, T.J.M.; Shinpaugh, J.L.; Varghese, S.L. KLL resonant transfer and excitation to $F^{6+}\left(1 s 2 l 2 l^{\prime}\right)$ intermediate states. Phys. Rev. A 1991, 44, 1636-1643. [CrossRef] [PubMed]

34. Benis, E.P.; Zouros, T.J.M.; Gorczyca, T.W.; Zamkov, M.; Richard, P. Isoelectronic study of triply excited Li-like states. J. Phys. B 2003, 36, L341-L348. [CrossRef]

35. Závodszky, P.A.; Aliabadi, H.; Bhalla, C.P.; Richard, P.; Tóth, G.; Tanis, J.A. Superelastic scattering of electrons from highly charged ions with inner shell vacancies. Phys. Rev. Lett. 2001, 87, 033202. [CrossRef] [PubMed]

36. Alnaser, A.S.; Landers, A.L.; Pole, D.J.; Hossain, S.; Haija, O.A.; Gorczyca, T.W.; Tanis, J.A.; Knutson, H. Supereleastic scattering of electrons from metastable He-like $\mathrm{C}^{4+}$ and $\mathrm{O}^{6+}$ ions. Phys. Rev. A 2002, 65, 042709. [CrossRef]

37. Borovik, A., Jr.; Müller, A.; Schippers, S.; Bray, I.; Fursa, D. Electron impact ionization of ground-state and metastable $\mathrm{Li}^{+}$ions. J. Phys. B 2009, 42, 025203. [CrossRef]

38. Renwick, A.C.; Bray, I.; Fursa, D.V.; Jacobi, J.; Knopp, H.; Schippers, S.; Müller, A. Electron-impact ionization of $\mathrm{B}^{3+}$ ions. J. Phys. B 2009, 42, 175203. [CrossRef] 
39. Schlummer, T.; Marchuk, O.; Schultz, D.; Bertschinger, G.; Biel, W.; Reiter, D.; Textor-Team, T. Comparison of effective rate coefficients for high energy charge-exchange with measurements of the Rydberg series of $\mathrm{Ar}^{16+}$ at the tokamak TEXTOR. J. Phys. B 2015, 48, 144033. [CrossRef]

40. Cui, Z.; Morita, S.; Zhou, H.; Ding, X.; Sun, P.; Kobayashi, M.; Cui, X.; Xu, Y.; Huang, X.; Shi, Z.; et al. Enhancement of edge impurity transport with ECRH in the HL-2A tokamak. Nucl. Fusion 2013, 53, 093001. [CrossRef]

41. Liu, L.; Jakimovski, D.; Wang, J.G.; Janev, R.K. Electron capture and excitation in $\mathrm{H}^{+}$-He $\left(1 \mathrm{~s} 2 \mathrm{~s} ;{ }^{1,3} \mathrm{~S}\right)$ collisions. J. Phys. B 2012, 45, 225203. [CrossRef]

42. Trassinelli, M.; Prigent, C.; Lamour, E.; Mezdari, F.; Merot, J.; Reuschl, R.; Rozet, J.P.; Steydli, S.; Vernhet, D. Investigation of slow collisions for (quasi) symmetric heavy systems: what can be extracted from high resolution x-ray spectra. J. Phys. B 2012, 45, 085202. [CrossRef]

43. Nandi, T.; Oswal, M.; Kumar, S.; Jhingan, A.; Abhilash, S.; Karmakar, S. Radiative resonant energy transfer: A new excitation process of beam-foil interaction. J. Quant. Spectrosc. Radiat. Transf. 2012, 113, 783-788. [CrossRef]

44. Lin, Y.C.; Ho, Y. Quantum entanglement for two electrons in the excited states of helium-like systems. Can. J. Phys. 2014, 93, 646-653. [CrossRef]

45. Betz, H.D. Charge States and Charge-Changing Cross Sections of Fast Heavy Ions Penetrating Through Gaseous and Solid Media. Rev. Mod. Phys. 1972, 44, 465-539. [CrossRef]

46. Sayer, R. Semi-empirical formulas for heavy-ion stripping data. Rev. Phys. Appl. 1977, 12, $1543-1546$. [CrossRef]

47. Rozet, J.; StÃphan, C.; Vernhet, D. ETACHA: A program for calculating charge states at $\{$ GANIL\} energies. Nucl. Instrum. Methods Phys. Res. B 1996, 107, 67-70. [CrossRef]

48. Dmitriev, I.S.; Nikolaev, V.S. Semi-empirical method for the calculation of the equilibrium distribution of charges in a fast-ion beam. Sov. Phys. JETP 1965, 20, 409-415.

49. Shima, K.; Kuno, N.; Yamanouchi, M.; Tawara, H. Equilibrium Charge Fractions of Ions of Z = 4-92 Emerging From A Carbon Foil. At. Data Nucl. Data Tables 1992, 51, 173-241. [CrossRef]

50. Schiwietz, G.; Grande, P. Improved charge-state formulas. Nucl. Instrum. Methods Phys. Res. B 2001, 175-177, 125-131. [CrossRef]

51. Asimakopoulou, E.M. TARDIS (Transmitted chARge DIStribution); Technical Report; Institute of Nuclear and Particle Physics and Department of Physics, University of Athens: Athens, Greece, 2014.

52. Hvelplund, P. Energy Loss and Straggling of $100-500-\mathrm{keV}^{90} \mathrm{Th},{ }^{82} \mathrm{~Pb},{ }^{80} \mathrm{Hg}$ and ${ }^{64} \mathrm{Gd}$ in $\mathrm{H}_{2}$. Phys. Rev. A 1975, 11, 1921-1927. [CrossRef]

53. Andersen, L.H.; Bolbo, J.; Kvistgaard, P. State-selective dielectronic-recombination measurements for Heand Li-like carbon and oxygen ions. Phys. Rev. A 1990, 41, 1293-1302. [CrossRef] [PubMed]

54. Andersen, L.H.; Pan, G.Y.; Schmidt, H.T.; Badnell, N.R.; Pindzola, M.S. Absolute measurements and calculaitons of Dielectronic Recombination with metastable He- N, F, and Si ions. Phys. Rev. A 1992, 45, 7868-7875. [CrossRef] [PubMed]

55. Andersen, L.H.; Hvelplund, P.; Knudsen, H.; Kvistgaard, P. State-selective Dielectronic-Recombination measurements for He-like Oxygen ions in an electron cooler. Phys. Rev. Lett. 1989, 62, 2656. [CrossRef] [PubMed]

56. Kilgus, G.; Habs, D.; Schwalm, D.; Wolf, A.; Schuch, R.; Badnell, N.R. Dielectronic recombination from ground state of heliumlike carbon ions. Phys. Rev. A 1993, 47, 4859. [CrossRef] [PubMed]

57. Benis, E.P.; Zamkov, M.; Richard, P.; Zouros, T.J.M. Comparison of two experimental techniques for the determination of the $1 s 2 s^{3} S$ metastable beam fraction in energetic $\mathrm{B}^{3+}$ ions. Nucl. Instrum. Methods Phys. Res. B 2003, 205, 517-521. [CrossRef]

58. Strohschein, D.; Röhrbein, D.; Kirchner, T.; Fritzsche, S.; Baran, J.; Tanis, J.A. Nonstatistical enhancement of the $1 s 2 s 2 p^{4} P$ state in electron transfer in $0.5-1.0-\mathrm{MeV} / \mathrm{u} \mathrm{C}^{4,5+}+\mathrm{He}$ and $\mathrm{Ne}$ collisions. Phys. Rev. A 2008, 77, 022706. [CrossRef]

59. Benis, E.P.; Zouros, T.J.M. Determination of the $1 s 2 \ell 2 \ell^{\prime}$ state production ratios ${ }^{4} P^{o} /{ }^{2} P,{ }^{2} D /{ }^{2} P$ and ${ }^{2} P_{+} /{ }^{2} P_{-}$ from fast $\left(1 s^{2}, 1 s 2 s^{3} S\right)$ mixed-state He-like ion beams in collisions with $\mathrm{H}_{2}$ targets. J. Phys. B 2016, 49, 235202. [CrossRef]

60. Schiebel, U.; Doyle, B.L.; Macdonald, J.R.; Ellsworth, L.D. Projectile K x rays from Si ${ }^{12+}$ ions in the $1 \mathrm{~s} 2 \mathrm{~s}^{3} \mathrm{~S}_{1}$ metastable state incident on helium gas. Phys. Rev. A 1977, 16, 1089. [CrossRef] 
61. Terasawa, M.; Gray, T.J.; Hagmann, S.; Hall, J.; Newcomb, J.; Pepmiller, P.; Richard, P. Electron capture by and electron excitation of two-electron fluorine ions incident on helium. Phys. Rev. A 1983, 27, 2868-2875. [CrossRef]

62. Zamkov, M.; Aliabadi, H.; Benis, E.P.; Richard, P.; Tawara, H.; Zouros, T.J.M. Energy dependence of the metastable fraction in $B^{3+}\left(1 s^{2}{ }^{1} S, 1 s 2 s^{3} S\right)$ beams produced in collisions with solid and gas targets. Phys. Rev. A 2001, 64, 052702. [CrossRef]

63. Zamkov, M.; Benis, E.P.; Richard, P.; Zouros, T.J.M. Fraction of metastable $1 s 2 s^{3} S$ ions in fast He-like beams $(Z=5-9)$ produced in collisions with carbon foils. Phys. Rev. A 2002, 65, 062706. [CrossRef]

64. Benis, E.P.; Zamkov, M.; Richard, P.; Zouros, T.J.M. Technique for the determination of the $1 \mathrm{~s} 2 \mathrm{~s}^{3} S$ metastable fraction in two-electron ion beams. Phys. Rev. A 2002, 65, 064701. [CrossRef]

65. Lamour, E.; Gervais, B.; Rozet, J.P.; Vernhet, D. Production and transport of long-lifetime excited states in preequilibrium ion-solid collisions. Phys. Rev. A 2006, 73, 042715. [CrossRef]

66. Drake, G.W.F.; Victor, G.A.; Dalgarno, A. Two-Photon Decay of the Singlet and Triplet Metastable States of Helium-like Ions. Phys. Rev. 1969, 180, 25-32. [CrossRef]

67. Drake, G.W.F. Theory of Relativistic Magnetic Dipole Transitions: Lifetime of the Metastable $2{ }^{3} \mathrm{~S}$ State of the Heliumlike Ions. Phys. Rev. A 1971, 3, 908-915. [CrossRef]

68. Fischer, C.F.; Gaigalas, G. Note on the 2s 21 S 0 -2s2p 3 P 1 intercombination line of B II and C III. Phys. Scr. 1997, 56, 436. [CrossRef]

69. Fischer, C.F. Multiconfiguration Dirac-Hartree-Fock Calculations for Be-like Intercombination Lines Revisited. Phys. Scr. 2000, 62, 458. [CrossRef]

70. Madesis, I.; Dimitriou, A.; Laoutaris, A.; Lagoyannis, A.; Axiotis, M.; Mertzimekis, T.; Andrianis, M.; Harissopulos, S.; Benis, E.P.; Sulik, B.; et al. Atomic Physics with Accelerators: Projectile Electron Spectroscopy (APAPES). J. Phys: Conf. Ser. 2015, 583, 012014. [CrossRef]

71. Turner, B.R.; Rutherford, J.A.; Compton, D.M.J. Abundance of Excited Ions in $\mathrm{O}^{+}$and $\mathrm{O}^{2+}$ Ion Beams. J. Chem. Phys. 1968, 48, 1602-1608. [CrossRef]

72. Pedersen, E.H. Metastable-Atom Population of Fast, Neutral Helium Beams. Phys. Rev. Lett. 1979, 42, 440-443. [CrossRef]

73. Meyer, F.; Havener, C.; Phaneuf, R.; Swenson, J.; Shafroth, S.; Stolterfoht, N. Evidence for correlated double-electron capture in slow collisions of multicharged ions with He and H2. Nucl. Instrum. Methods Phys. Res. B 1987, 24-25, 106-110. [CrossRef]

74. Müller, A.; Borovik, A.; Buhr, T.; Hellhund, J.; Holste, K.; Kilcoyne, A.L.D.; Klumpp, S.; Martins, M.; Ricz, S.; Viefhaus, J.; Schippers, S. Near-K-edge single, double, and triple photoionization of $\mathrm{C}^{+}$ions. Phys. Rev. A 2018, 97, 013409. [CrossRef]

75. Cocke, C.L.; Varghese, S.L.; Curnutte, B. Yields of K-vacancy-bearing metastable states following foil excitation. Phys. Rev. A 1977, 15, 874. [CrossRef]

76. Welton, R.F.; Moran, T.F.; Thomas, E.W. Metastable state abundances in multiply charged ion beams. J. Phys. B 1991, 24, 3815. [CrossRef]

77. Bliek, F.W.; Hoekstra, R.; Bannister, M.E.; Havener, C.C. Low-energy electron capture by $\mathrm{C}^{4+}$ ions from atomic hydrogen. Phys. Rev. A 1997, 56, 426. [CrossRef]

78. Müller, A.; Borovik, A.; Huber, K.; Schippers, S.; Fursa, D.V.; Bray, I. Double-K-vacancy states in electronimpact single ionization of metastable two-electron $\mathrm{N}^{5+}\left(1 s 2 s{ }^{3} \mathrm{~S}_{1}\right)$ ions. Phys. Rev. A 2014, 90, 010701. [CrossRef]

79. Meyerhof, W.E. K-Vacancy Sharing in Near-Symmetric Heavy-Ion Collisions. Phys. Rev. Lett. 1973, 31, 1341-1344. [CrossRef]

80. Benis, E.P.; Doukas, S.; Zouros, T.J.M. Evidence for the non-statistical population of the $1 s 2 s 2 p^{4} P$ metastable state by electron capture in $4 \mathrm{MeV}$ collisions of $B^{3+}\left(1 s 2 s^{3} S\right)$ with $\mathrm{H}_{2}$ targets. Nucl. Instrum. Methods Phys. Res. B 2016, 369, 83-86. [CrossRef]

81. Doerfert, J.; Träbert, E.; Wolf, A.; Schwalm, D.; Uwira, O. Precision Measurement of the Electric Dipole Intercombination Rate in $C^{2+}$. Phys. Rev. Lett. 1997, 78, 4355-4358. [CrossRef]

82. Träbert, E.; Wolf, A.; Gwinner, G. Measurement of EUV intercombination transition rates in Be-like ions at a heavy-ion storage ring. Phys. Lett. A 2002, 295, 44-49. [CrossRef] 
83. Müller, A.; Schippers, S.; Phaneuf, R.A.; Kilcoyne, A.L.D.; Bräuning, H.; Schlachter, A.S.; Lu, M.; McLaughlin, B.M. Fine-structure resolved photoionization of metastable Be-like ions C III, N IV, and O V. J. Phys. Conf. Ser. 2007, 58, 383-386. [CrossRef]

84. Stolterfoht, N.; Miller, P.D.; Krause, H.F.; Yamazaki, Y.; Swenson, J.K.; Bruch, R.; Dittner, P.F.; Pepmiller, P.L.; Datz, S. Surgery of fast, highly charged ions studied by zero-degree Auger spectroscopy. Nucl. Instrum. Methods Phys. Res. B 1987, 24/25, 168-172. [CrossRef]

85. Scully, S.W.J.; Aguilar, A.; Emmons, E.D.; Phaneuf, R.A.; Halka, M.; Leitner, D.; Levin, J.C.; Lubell, M.S.; Püttner, R.; Schlachter, A.S.; et al. K-shell photoionization of Be-like carbon ions: experiment and theory for $\mathrm{C}^{2+}$. J. Phys. B 2005, 38, 1967-1975. [CrossRef]

86. Shorman, M.M.A.; Gharaibeh, M.F.; Bizau, J.M.; Cubaynes, D.; Guilbaud, S.; Hassan, N.E.; Miron, C.; Nicolas, C.; Robert, E.; Sakho, I.; et al. K-shell photoionization of Be-like and Li-like ions of atomic nitrogen: experiment and theory. J. Phys. B 2013, 46, 195701. [CrossRef]

87. Müller, A.; Schippers, S.; Phaneuf, R.A.; Scully, S.W.J.; Aguilar, A.; Cisneros, C.; Gharaibeh, M.F.; Schlachter, A.S.; McLaughlin, B.M. K-shell photoionization of Be-like boron ( $\left.\mathrm{B}^{+}\right)$ions: experiment and theory. J. Phys. B 2014, 47, 135201. [CrossRef]

88. McLaughlin, B.M.; Bizau, J.M.; Cubaynes, D.; Guilbaud, S.; Douix, S.; Shorman, M.M.A.; Ghazaly, M.O.A.E.; Sakho, I.; Gharaibeh, M.F. K-shell photoionization of $\mathrm{O}^{4+}$ and $\mathrm{O}^{5+}$ ions: experiment and theory. MNRAS 2017, 465, 4690-4702. [CrossRef]

89. Benis, E.P.; Madesis, I.; Laoutaris, A.; Nanos, S.; Zouros, T.J.M. Experimental determination of the effective solid angle of long-lived projectile states in zero-degree Auger projectile spectroscopy. J. Electron Spectrosc. Relat. Phenom. 2018, 222, 31-39. [CrossRef]

90. Doukas, S.; Madesis, I.; Dimitriou, A.; Laoutaris, A.; Zouros, T.J.M.; Benis, E.P. Determination of the solid angle and response function of a hemispherical spectrograph with injection lens for Auger electrons emitted from long lived projectile states. Rev. Sci. Instrum. 2015, 86, 043111. [CrossRef] [PubMed]

91. Benis, E.; Doukas, S.; Zouros, T.; Indelicato, P.; Parente, F.; Martins, C.; Santos, J.; Marques, J. Evaluation of the effective solid angle of a hemispherical deflector analyser with injection lens for metastable Auger projectile states. Nucl. Instrum. Methods Phys. Res. B 2015, 365, 457-461. [CrossRef]

92. SIMION 8.1.3.4; Scientific Instrument Services, Inc.: Ringoes, NJ, USA, 2017. Available online: http: / / www.simion.com (accessed on 01 April 2017).

93. Zamkov, M.; Aliabadi, H.; Benis, E.P.; Richard, P.; Tawara, H.; Zouros, T.J.M. Absolute cross sections and decay rates for the triply excited $B^{2+}\left(2 s 2 p^{2} \mathrm{D}\right)$ resonance in electron-metastable-ion collisions. Phys. Rev. A 2002, 65, 032705. [CrossRef]

94. Zouros, T.J.M.; Benis, E.P.; Gorczyca, T.W.; González, A.D.; Zamkov, M.; Richard, P. Differential electron scattering from positive ions measured by zero-degree ion-atom spectroscopy. Nucl. Instrum. Methods Phys. Res. B 2003, 205, 508-516. [CrossRef]

95. Schneider, D.; Bruch, R.; Butscher, W.; Schwarz, W.H.E. Prompt and time-delayed electron decay-in-flight spectra of gas-excited carbon ions. Phys. Rev. A 1981, 24, 1223-1236. [CrossRef]

96. Zouros, T.J.M.; Sulik, B.; Gulyás, L.; Tökési, K. Selective enhancement of $1 s 2 s 2 p^{4} P_{J}$ metastable states populated by cascades in single-electron transfer collisions of $F^{7+}\left(1 s^{2} / 1 s 2 s^{3} S\right)$ ions with $\mathrm{He}$ and $\mathrm{H}_{2}$ targets. Phys. Rev. A 2008, 77, 050701. [CrossRef]

97. Lamour, E.; Fainstein, P.D.; Galassi, M.; Prigent, C.; Ramirez, C.A.; Rivarola, R.D.; Rozet, J.P.; Trassinelli, M.; Vernhet, D. Extension of charge-state-distribution calculations for ion-solid collisions towards low velocities and many-electron ions. Phys. Rev. A 2015, 92, 042703. [CrossRef]

98. Gao, J.W.; Wu, Y.; Sisourat, N.; Wang, J.G.; Dubois, A. Single- and double-electron transfer in low- and intermediate-energy $\mathrm{C}^{4+}+$ He collisions. Phys. Rev. A 2017, 96, 052703. [CrossRef]

99. Gao, J.W.; Wu, Y.; Wang, J.G.; Sisourat, N.; Dubois, A. State-selective electron transfer in $\mathrm{He}^{+}+\mathrm{He}$ collisions at intermediate energies. Phys. Rev. A 2018, 97, 052709. [CrossRef]

(C) 2018 by the authors. Licensee MDPI, Basel, Switzerland. This article is an open access article distributed under the terms and conditions of the Creative Commons Attribution (CC BY) license (http:/ / creativecommons.org/licenses/by/4.0/). 